\title{
Interactive comment on "Relating Climate Sensitivity Indices to projection uncertainty" by Benjamin Sanderson
}

\section{Ben Sanderson}

sanderson@cerfacs.fr

Received and published: 9 March 2020

Thanks to the reviewer for thorough reading and thoughtful points. I've endeavoured to address the issues raised in the revised paper as detailed below.

\section{Major Issues}

1)... the model consists of Eqs. B1 and B2, together with B3 for including a transient forcing. An optimization procedure is applied to estimate the parameters based on a given data set (HadCRUT) and cost functions (B4-B6). I do not completely understand how this optimization defines the parameter distribution (the model ensemble),

I have expanded the discussion of what the MCMC optimization does and how the 
i.e. how is the distribution exactly derived from the optimization, and how do(es) the distribution(s) look(s) like (a Figures of the pdfs may be helpful in this respect)?

The relevant figure is in the supplemental material, Figure S3 - which shows the indi-

Interactive vidual and pairwise parameter distributions of the posterior ensemble.

comment

Furthermore (random order):

(i) in L210 it is stated that CO2 concentrations enter the cost functions, but $H(t)$ and $D(t)$ seem to be heat fluxes (L215)?

Sorry - that was a typo, the treatment of $\mathrm{CO} 2$ is specific to the companion paper to this one, which included carbon cycle feedbacks. This paper only considers the thermal part of the model. Typo corrected.

(ii) How do $H$ and $D$ relate to the parameters $r 1, r 2$ in $B 2$ (are they the same)?

Now included specific equations for $D$ and $H$

(iii) Are $T \_p(B 3)$ and $P(B 1)$ the same?

Yes, now T_p throughout

(iv) How does $F(B 3)$ relate to $R(B 2)$ (or how are eq. B1 and eq. B2 coupled in the model).

The coupling is in the original multi-timescale energy balance model as detailed in Millar et al 2017 (now written explicitly). The particular solutions of the temperature and

Printer-friendly version

Discussion paper 
radiative response to a step change in forcing can be written as a sum of exponential decays (again, now shown explicitly)

ESDD

(v) How (where) does the non CO2 forcing factorf_r(L208) enters the equations.

Now included an explicit expansion of the historical forcing timeseries $F(t)$ which defines $f \_r$

2) I'm wondering how important the non-CO2 forcing agents (L207) and the factor f_r are for the results. How much of the variability of the control (present day) climate is explained by the non-CO2 forcing, and how is the non-CO2 forcing prescribed in the scenarios (it seems that all is represented by a constant $f \_r$ )?

The constant $f \_r$ is a scaling factor (as now made clear by equation B5), so the non$\mathrm{CO} 2$ forcing is not constant over time - but you are correct that we are assuming that there is only a single degree of freedom in optimization. Though we could break down this forcing further - our primary goal is not to attribute the response to different forcings, and this formulation allows conceptually for uncertainty in the historical forcing timeseries while minimizing the number of degrees of freedom in the optimization.

3) Not much attempt is made to evaluate/validate the models behaviour under RCP scenarios. So far (as far as I can see) it is only shown that the model reasonably reproduces the HadCRUT data (where it is constrained to), and gives a response within the $C M I P$ range. It would be useful to show that the model can reasonably reproduce the RCP8.5/RCP2.6 response of one particular model if the parameters are constrained by the present day simulation of the same model. This would give more confidence to the obtained results.

I've included an additional supplementary plot to fit the pulse-response model to historical simulations in the CMIP archive with future ensemble projections for RCP2.6 and
Interactive comment
Printer-friendly version

Discussion paper 
8.5, with some discussion in the methods. The technique generally performs well (i.e. future projections fall within the distribution), with the exception of a couple of models which show little or no long term warming response (CCSM4, FGoals, FIO-ESM - models which share some fraction of their codebase). I've added a caveat to this effect, but I'm broadly happy that the technique is producing reasonable probabilistic fits to historical CMIP data.

ESDD

Interactive comment

4) One main result is that residual drift may explain 'surprising' results regarding EffCS and TCR in CMIP. From Fig. 3 we see that different CMIP models seem to exhibit different magnitudes of residual drift. I'm wondering whether the simple model result regarding the effect of drift can be qualitatively checked by comparing respective simulations.

l've attempted to show this qualitatively in a new Figure 5, and supplementary Figure S5. The former is an attempt to 'correct' the control drift uncertainty in the estimation of TCR by estimating baseline temperatures from the 1 pctCO2 simulation itself, and background trends from the control simulation. The plots show - for all 3 metrics, but particularly for TCR - that the correlation with 21st century warming under RCP8.5 can be improved a little using this baseline correction, supporting the hypothesis that control drift is an issue for the estimation of sensitivity metrics. Of course this is just an estimate - itself noisy given the relatively short regression, which is noted in the text at the end of the results section, but the improvement over the PIControl average is notable.

\section{Some Minor}

1) A common question concerning studies utilizing such simplified models is the sensitivity to the particular choice of the model setup. In this respect, the author may like to comment on the sensitivity of the results with respect to the particular choice of the

Printer-friendly version

Discussion paper 
number of timescales ( $n=2$ in B1 \& B2). How different would be the results for $n=1$ (or $n=3)$ ?

ESDD

Repeating the entire analysis with a different model dimensionality is beyond scope, but during development, I experimented with different timescales dimensions - 1 timescale can be trivially dismissed as unable to represent the temporal evolution of the models in response to $4 \times \mathrm{CO} 2$ forcing. Beyond two timescales, only slight improvement is seen in the fitting error - so two timescales was chosen for this study to be (a) consistent with existing literature (i.e. within the framework of FAIR, which is in common usage), (b) lower dimensional so easier to interpret in terms of slow/deep ocean and fast/shallow ocean response and (c) applicable to CMIP models in.

Fundamentally - only some models show a slightly improved fit with an extra allowed timescale (see GISS-H, for example on the below plot). Other models are adequately described with 2 , and adding a 3rd results in a degenerate fit. Thus - a cross ensemble analysis of the additioanl degree of freedom is not clearly defined. Other studies have arrived at the same conclusion (see Proistosescu and Huybers http://doi.org/10.1126/sciadv.1602821, Smith 2018 http://dx.doi.org/10.5194/gmd-112273-2018, Geoffroy 2012 http://doi.org/10.1175/JCLI-D-12-00196.1 ).

Ultimately, for this study, the aim is to reproduce the basic features of CMIP ensemble diversity in response to different types of forcing with the minimum possible complexity of model - and I felt that this was both possible and easier to explain with the two timescale model. Clearly, the real world could have the capacity to respond to forcing on a range of timescales, but two timescales adequately describe the response to forcing on the century timescale in the CMIP ensemble.

I have added a paragraph in the conclusions on how the structural assumption of two timescales may impact results. Primarily - I think the current method is support the primary conclusions that ECS and TCR are insufficient to constrain some future warming

Interactive comment
Printer-friendly version

Discussion paper 
trends (i.e. ECS or TCR are insufficient to describe RCP2.6 warming), and that nonequilibration is a problem for measurement. But I do agree that the structural assumption of 2 timescales might impact the constraint, for example, of ECS from historical temperatures - and l've added this caveat.

2) The author introduces a new metric (A140) as an alternative. It would be useful if the Interactive author could illustrate the behaviour of $A 140$ (in contrast to EFFCS) for a CMIP data set.

A140 is included in new Figure 5, and estimated values are included in new Table 2.

3) In the abstract, the author quantifies the relative errors for T140 and EFFCS in the simple model framework. As these numbers may certainly not be the same for CMIP model, the may not be part of the abstract.

I have removed these quantitative results from the abstract accordingly

4) f_r appears twice in Table B1

Fixed

Interactive comment on Earth Syst. Dynam. Discuss., https://doi.org/10.5194/esd-2019-77, 2019. 


\section{ESDD}

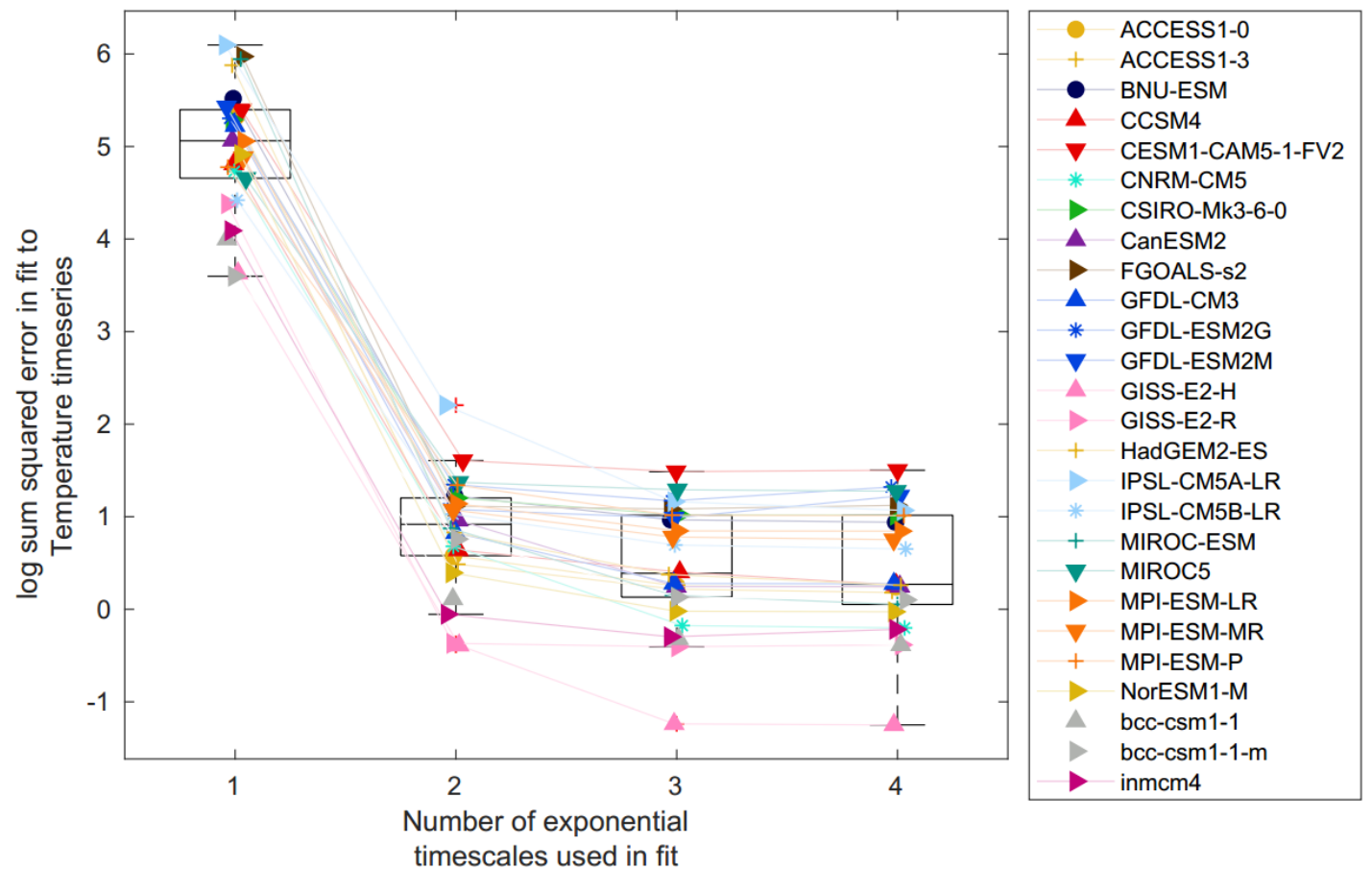

Interactive
comment

Fig. 1. 